\title{
Phase space vortices in collisionless plasmas
}

\author{
P. Guio ${ }^{1}$, S. Børve ${ }^{1}$, L. K. S. Daldorff ${ }^{1}$, J. P. Lynov ${ }^{2}$, P. Michelsen ${ }^{2}$, H. L. Pécseli ${ }^{3}$, J. Juul Rasmussen ${ }^{2}$, K. Saeki ${ }^{4}$, and \\ J. Trulsen ${ }^{1}$ \\ ${ }^{1}$ University of Oslo, Institute for Theoretical Astrophysics, Box 1029 Blindern, N-0315 Oslo, Norway \\ ${ }^{2}$ Risø National Laboratory, Department of Optics and Fluid Dynamics, DK-4000 Roskilde, Denmark \\ ${ }^{3}$ University of Oslo, Physics Department, Box 1048 Blindern, N-0316 Oslo, Norway \\ ${ }^{4}$ Department of Physics, Shizuoka University, Ohya 836, Shizuoka 422-8529, Japan
}

Received: 01 October 2001 - Revised: 12 February 2002 - Accepted: 9 April 2002

\begin{abstract}
Results on the formation and propagation of electron phase space vortices from laboratory experiments are summarized. The electron phase space vortices were excited in a strongly magnetized Q-machine plasma by applying a pulse to a segment of a waveguide surrounding the plasma. Depending on the temporal variation of the applied pulse, one or more phase space vortices can be excited, and their interaction can be followed in space and time. We were able to demonstrate, for instance, an irreversible coalescence of two such vortices. These results are extended by numerical simulations, showing how electron phase space vortices can also be formed by beam instabilities. Furthermore, a study of ion phase space vortices is performed by numerical simulations. Both codes allow for an externally applied magnetic field in three spatial dimensions. Ion phase space vortices are formed by the nonlinear saturation of the ion-ion two-stream instability, excited by injecting an ion beam at the plasma boundary. By following the evolution of the ion distribution of the velocity perpendicular to the direction of propagation of the injected ion beam, we find a significant ion heating in the direction perpendicular to the magnetic field associated with the ion phase space vortices being formed. The results are relevant, for instance, for the interpretation of observations by instrumented spacecraft in the Earth's ionosphere and magnetosphere.
\end{abstract}

\section{Introduction}

Analytical studies demonstrated that collisionless plasmas, as described by the Vlasov equation, formally allow for a large class of nonlinear equilibrium states, BGK-modes. The original studies referred to one spatial dimension (Bernstein et al., 1957), but later these results were also generalized to two and three spatial dimensions, although with imposed symmetry conditions (Kato, 1976; Ishibashi and Kitahara, 1992). Theoretically, the phase space dynamics of electrons

Correspondence to: H. L. Pécseli (hans.pecseli@ fys.uio.no) and ions can support a surprisingly rich variety of electrostatic structures. In reality, relatively few types are observed; here we shall only be concerned with the so-called phase space vortices. The formation of such states was first observed in numerical simulations of electron beam instabilities (Roberts and Berk, 1967; Berk et al., 1970), and later in a series of laboratory experiments. The phenomena discussed here are uniquely related to the phase space dynamics of collisionless plasmas, and thus represent kinetic phenomena, which cannot be recovered by simple fluid models.

Phase space vortices are often formed as the saturated nonlinear stage of beam instabilities, or by sudden disturbances, often externally imposed on the plasma. The processes determining the lifetime of phase space vortices in one, two and three spatial dimensions is not well understood: in studies of the nonlinear evolution of electron two-stream instabilities, Morse and Nielson (1969) found that electron vortices had a very long lifetime in one dimension, disintegrated slowly in two spatial dimensions and disappeared quite rapidly in three spatial dimensions for unmagnetized plasmas, although not much was learned concerning the nature of the processes which lead to the destruction of the phase space vortices. The dynamics in two or three spatial dimensions are for several reasons, distinct from one spatial dimensions (Pécseli, 1987). It might be that an externally imposed magnetic field prolongs the vortex lifetime, but numerical simulations of electron vortices (Oppenheim et al., 1999) also show, in this case, a breakup of vortex structures, at least for the parameters investigated there. We recall here that the experimental observation of electron vortices reported by Saeki et al. (1979) were actually obtained in magnetized plasmas, with very strong magnetic fields, $\omega_{c e}>\omega_{p e}$, with $\omega_{c e}$ and $\omega_{p e}$ being the electron cyclotron and the electron plasma frequency, respectively.

In the present study we first summarize, in Sect. 2, some results concerning electron phase space vortices, also called "electron holes", from the original experiment of Saeki et al. (1979). This section serves as a brief review of the experimental results, but shows data that have not been published 


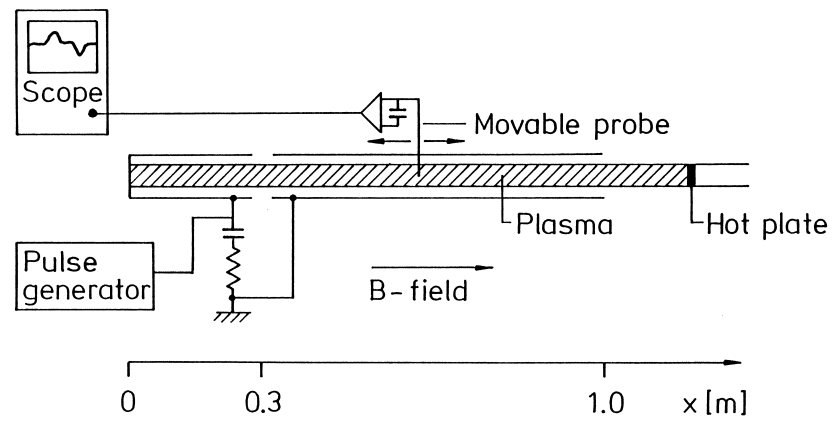

Fig. 1. Schematic diagram for the experimental setup for the excitation of electron phase space vortices.

before. In Sect. 3, we present new results concerning the ion phase space counterpart of the electron holes. These results are obtained by a fully three-dimensional particle-incell simulation of low-frequency electrostatic phenomena in magnetized plasmas. In Sect. 4, we discuss the relation of our results to in situ observations made by instrumented spacecrafts. Finally, Sect. 5 contains our conclusions. Our emphasis in the present study is on phase space vortices. Also shock phenomena are conspicuous in the summarized experimental, as well as numerical results. Concerning these features, we refer to selected publications.

\section{Electron phase space vortices}

Electron phase space vortices can be generated in a controlled laboratory experiment by applying an electric field across a gap in a cylindrical waveguide containing the plasma column in a strongly magnetized plasma. The first experimental results were reported by Saeki (1973), with detailed experimental observations carried out later in a strongly magnetized plasma in the Risø Q-machine by Saeki et al. (1979). A detailed discussion, including also numerical simulations, was later presented by Lynov et al. (1979) (see also a review by Schamel, 1986). A somewhat simplified numerical simulation, based on a "water-bag" model, was shown by Saeki et al. (1979). Albeit simplified, these simulations may provide an illustrative visualization of the formation process.

\subsection{Experimental results}

The experimental set up is shown in Fig. 1. The basic parts of the circuit for the excitation with a pulse generator are shown, with positions marked along the magnetized plasma column. The pulses propagate to the right, in the upstream direction of the plasma. In electron rich conditions with negative endplate bias (Motley, 1975), the electron mean velocity is almost vanishing, while the ions have a net drift away from the hot plate. For the short time scales of the experiment, the ions can be considered as an immobile background of positive charge, and the net ion flow is immaterial for the interpretation of the observations. Basic results from the experiment were shown by Saeki et al. (1979) and Lynov et al. (1979) for fixed probe positions and signals varying with time. Here, in Fig. 2, we show the spatial variation for selected times, allowing for a direct comparison with numerical simulations (Lynov et al., 1979). These results have not been shown before. We note a perturbation propagating at a high speed, followed by a structure propagating with a constant velocity, which is slightly below the electron thermal velocity. The fast structure can develop into a shock or a soliton-like perturbation, depending on the time variation of the externally applied signal. The properties of the shock were investigated by Saeki and Ikezi (1972) and Saeki (1973), while the soliton was studied by Karpman et al. (1979).

A comparison of the experimental observations with the numerical results presented by Lynov et al. (1979) shows a remarkable agreement, as far as the space-time variation of the electrostatic potential is concerned. The agreement is particularly conspicuous when the presentation in Fig. 2 is used. We can safely conclude that the phase space variation in simulations and the experiment is also in agreement. The electron phase space vortex represents a local deficit of electrons, and is thus associated with a net positive charge. The resulting positive potential "hump" can trap a fraction of the electron population, giving rise to a vortex-like structure when observed in phase space, hence giving it the name. Since the structure is associated with a local depletion in electron phase space, it is sometimes also called an "electron hole". The excitation requires relatively large applied voltages, in excess of $2 \mathrm{~V}$, where we operated with amplitudes up to $20 \mathrm{~V}$. However, the potential in the plasma was noticeably smaller than the applied one, and we estimate the minimum amplitude imposed variation in the plasma potential to be of the order of $0.3 \mathrm{~V}$ for this particular type of vortex excitation. The potential energy of an electron at this amplitude turns out to be of the same order of magnitude as the electron thermal energy.

Numerical studies by Saeki et al. (1979) and Lynov et al. (1979) also gave detailed insight into the excitation mechanism in the experiment. These studies make it clear that the excitation cannot be interpreted as being caused by an electron beam injected into the upstream plasma, facing the hot cathode in Fig. 1. It is due rather to particle trapping in the combined electrostatic fields from the applied pulse, and those induced by the perturbed charge distributions in the plasma. In the experiment, the electron holes are formed, together with the electron shocks, but this is a consequence of the particular excitation mechanism: it is quite possible to excite electron holes alone, as evidenced, for instance, by numerical simulations.

After formation, the electron hole retains its shape with an almost negligible deformation. Within the parameter variations we could achieve in the experiment, we did not find any systematic relation between the hole amplitude and velocity. According to the analytical results for the fully developed, nonlinear BGK-structures discussed here (Bernstein et al., 1957), there should be none. In the experiment, we easily obtained potential values $e \phi / T_{e}$ up to 1.5 , and in order to have a good signal-to-noise ratio, most of our observations are for relatively large amplitudes. We did not observe 


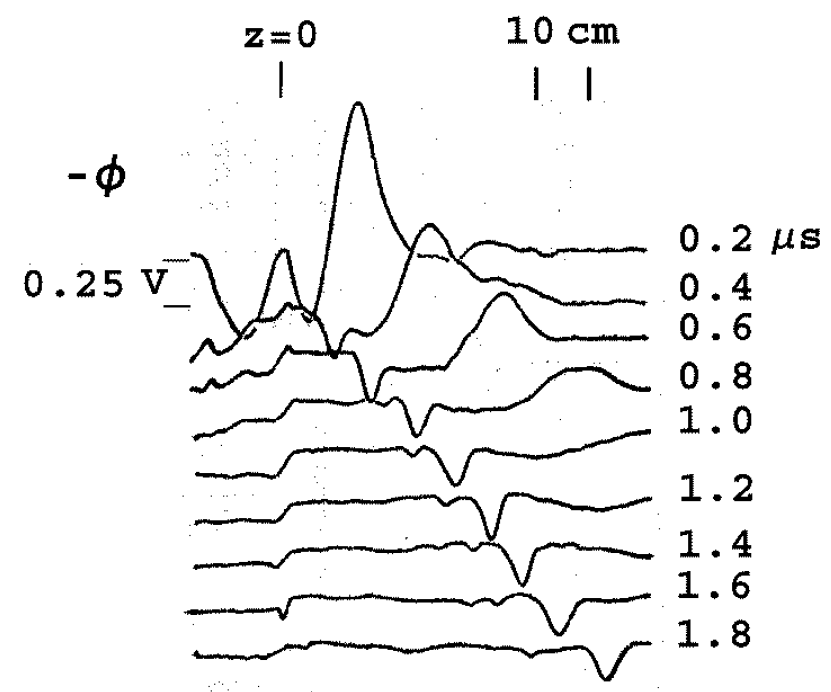

Fig. 2. Spatial variation of the electrostatic potential for selected times. The results were obtained by use of a sampling oscilloscope, set at a fixed time delay. The electron hole corresponds here to a potential depletion, since the polarity of the potential is reversed in the figure.

any relationship between hole amplitude and hole width either. The observed hole widths in the direction parallel to $\mathbf{B}$ were typically $15-20 \lambda_{D e}$, where $\lambda_{D e}$ is the electron Debye length. In the B-transverse direction, the hole width was determined by the diameter of the plasma column (see also results by lizuka and Tanaca, 1986). A simplified water-bag model for the study of the range of parameter variation was presented by Lynov et al. (1985), and it turns out to account for many of the basic properties, where the radial boundary conditions in the experiment are also of some importance. A more general kinetic model was used by Schamel (1979).

One conspicuous feature associated with the early numerical simulations (Berk et al., 1970) showing the formation of phase space vortices, was the seemingly irreversible coalescence of two such structures. By careful shaping of the exciter pulse in the experiment, we were able to excite two spatially separated electron holes with slightly different velocities, and to also observe here their subsequent coalescence. An illustration of this phenomenon is given in Fig. 3. The resulting electron hole is wider and has a larger amplitude than either of its constituents, with a velocity somewhere intermediate between their two velocities, although these details are seen most clearly in numerical simulations. Electron holes need not, however, always coalesce. In some numerical simulations (Lynov et al., 1979), it was found that they could pass through each other in case that their relative velocity was large. A numerical parameter study separating regions of coalescence from the case where holes pass through each other was presented by Lynov et al. (1980). The conclusion of this investigation was that the two important parameters are the relative velocities between the two phase space vortices, and the sum of their two peak potential amplitudes.

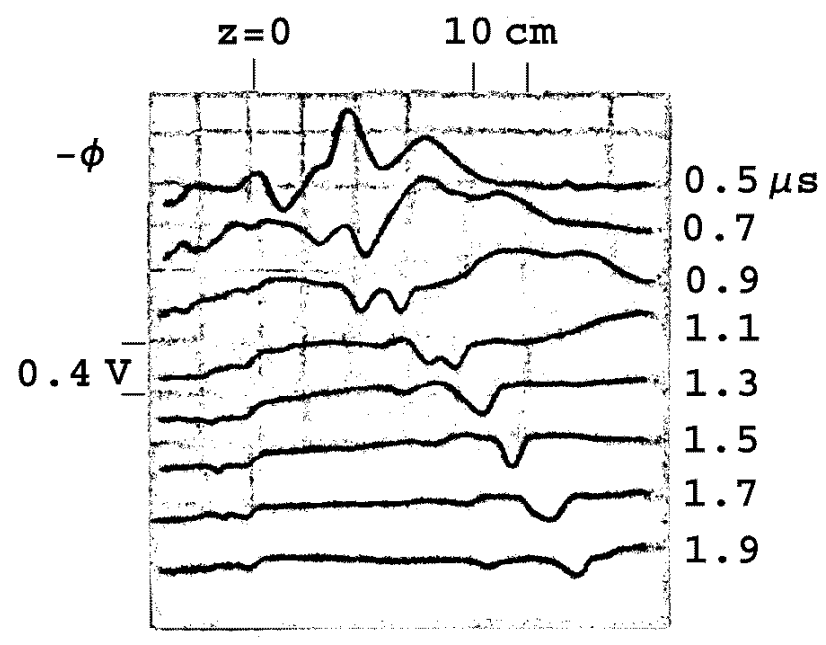

Fig. 3. Coalescence of two initially separated electron holes. The figure shows spatial variations for different times, obtained as in Fig. 2.

Small relative velocities lead to coalescence, but large amplitude structures tended to coalesce even when the relative velocities were large. The range of parameter variations in the study of Lynov et al. (1980) was, however, limited, and the problem deserves further scrutiny.

\subsection{Numerical results}

The investigations of Lynov et al. (1979) dealt with one electron population in a background of uniformly distributed, immobile ions in a one-dimensional model. The plasma was subsequently perturbed by an externally applied electric field. In the present study, we show results from a different numerical simulation where the excitation is accomplished by an external beam injection. Related simulations in one spatial dimension were performed by Singh and Schunk (1984). Our simulations were performed in a fully threedimensional magnetized plasma, using $27 \cdot 10^{6}$ simulation particles. We use floating boundary conditions for the electrostatic potential in such a way that the normal component of the electric field is vanishing there, corresponding to a Neumann problem. When dealing with the particles, we distinguish between two species: the beam and the background. The latter is initially uniformly distributed over the entire configuration space, having a Maxwellian velocity distribution, with temperature $T_{e}$, which we also use for reference. Background particles are injected every time one leaves the system. The position of any injected particle is randomly chosen in the box-surface through which the particle is leaving, so that the net particle flux through the surface is conserved. The velocity of the injected particles is chosen randomly from a population with the initial temperature. The beam particles are injected at one boundary with a velocity component along the magnetic field lines. The positions at 

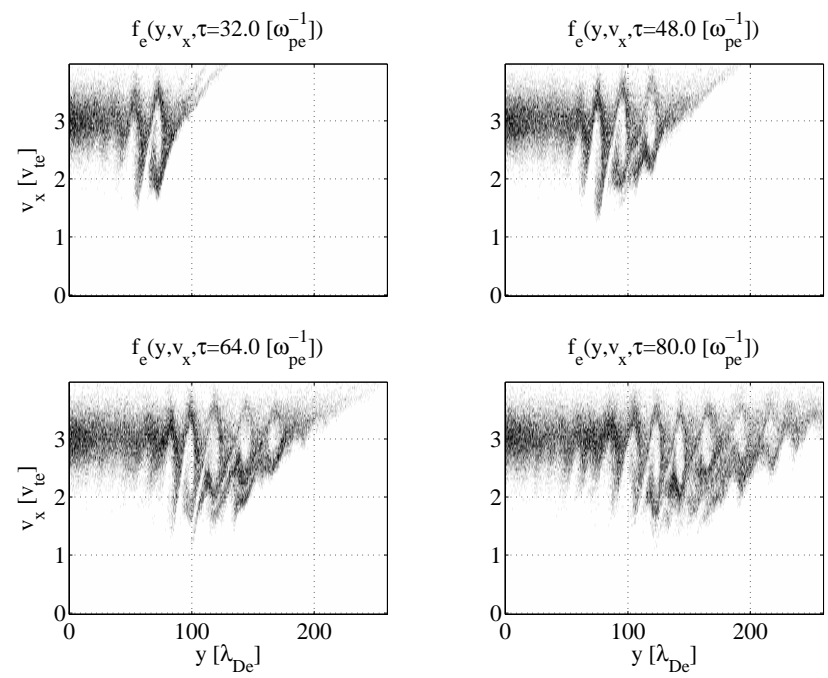

Fig. 4. Electron phase space vortices, or electron holes, formed by injection of an electron beam along the $y$-axis, into a stationary background. The phase space for the beam particles is shown. The figure is obtained as the average of particles in a region of $10 \times$ $10 \lambda_{D e}^{2}$ in the $(x, z)$-plane, at the center of the beam region. We have a magnetic field corresponding to $\omega_{c e} / \omega_{p e}=0.25$. The beam density is $20 \%$ of the background density.

the corresponding surface are chosen according to a Gaussian distribution in each direction, allowing for an elliptical cross section of the beam. The velocity of any particle is chosen randomly from a population in such a way that their flux corresponds to that of a Maxwellian having a net drift. A beam particle is lost when it leaves the simulation region. After injection at the boundary, the particle velocity and position evolves self-consistently. The ion component here is treated as an immobile background of uniform positive charge. Basically, our code is standard (Birdsall and Langdon, 1985). Numerical investigations by Singh et al. (2000) also allow for the ion motion, assuming a mass ratio corresponding to electrons and hydrogen ions, and studies an extended time evolution.

As an illustration, we use an electron beam density of $20 \%$ of the background electron density. The beam temperature is $T_{b}=0.1 T_{e}$. The cross section of the electron beam is elliptical, with aspect ratio 0.5 . The major axis is $43 \%$ of the $x$-dimension of the simulation box, where we use $46 \times 46 \lambda_{D e}^{2}$ in the $(x, z)$-plane, and $260 \lambda_{D e}$ along the $y$-direction, which is parallel to $\mathbf{B}$.

Electron holes are formed by injection of the electron beam along the magnetic field lines, just as in the simulations by Morse and Nielson (1969), although this investigation dealt with an initial condition problem in unmagnetized plasmas. Illustrative results are shown in Figs. 4 and 5. The corresponding potential variations (not shown here) demonstrate that the vortices are associated with local potential maxima, as expected. We note that the number of electron vortices is increasing with time, as the beam propagates into the plasma. With the present conditions, the vortex ve-
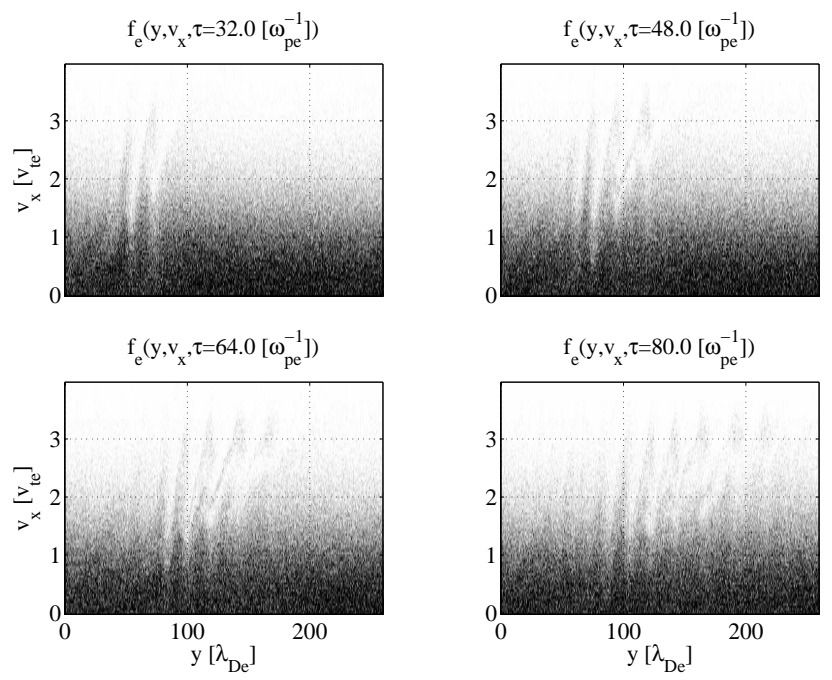

Fig. 5. Electron phase space for the background electron component, corresponding to Fig. 4.

locities are relatively close to that of the beam. An increase in the beam density results in a reduction of the vortex velocity. For the results shown in Figs. 4 and 5, the coupling to the background electron population is relatively small, and these electrons participate only marginally in the vortex formation. For smaller vortex velocities, this coupling is enhanced, as expected.

We varied the average injected electron velocity, $u_{b}$, and found a significant variation in the characteristic electron hole width, $\Delta$ (see Fig. 6). For velocities $\sim 2 \sqrt{T_{e} / m} \equiv 2 v_{t h}$ or smaller, we found the plasma to be stable for the present parameters, while electron holes were formed for all injection velocities larger than this. The upper limit of beam velocity was restricted by the requirement that the propagation time through the given simulation box should be sufficient for the formation of an electron hole. In Fig. 6, the magnetic field intensity was chosen so that $\omega_{c e} / \omega_{p e}=0.5$, but our results apply to a wide range of magnetic fields. We note that, within the uncertainty, the electron hole width increases approximately in proportion to the beam velocity. In this context, we recall that for a low density, high velocity beam injected into a stationary plasma, the linearly, most unstable wavelength of the excited electron plasma waves is approximately $u_{b} / \omega_{p e}=\left(u_{b} / v_{t h}\right) \lambda_{D e}$. Apart from a factor of $\sim 2$, this length is close to the observed $\Delta$, and has the same velocity scaling. Indirectly, we thus have indications for a relation between electron hole formation in beam systems and the linear instability, as anticipated by, for example, Kako et al. (1971).

We investigated the short time stability of electron holes for varying magnetic fields. In view of the observations of Morse and Nielson (1969), we found individual electron holes to be perhaps surprisingly stable. A magnetic field improved their stability. For instance, with $u_{b} / v_{t e}=3$, we found that for $\omega_{c e}>\omega_{p e} / 4$, the formation and time evolu- 


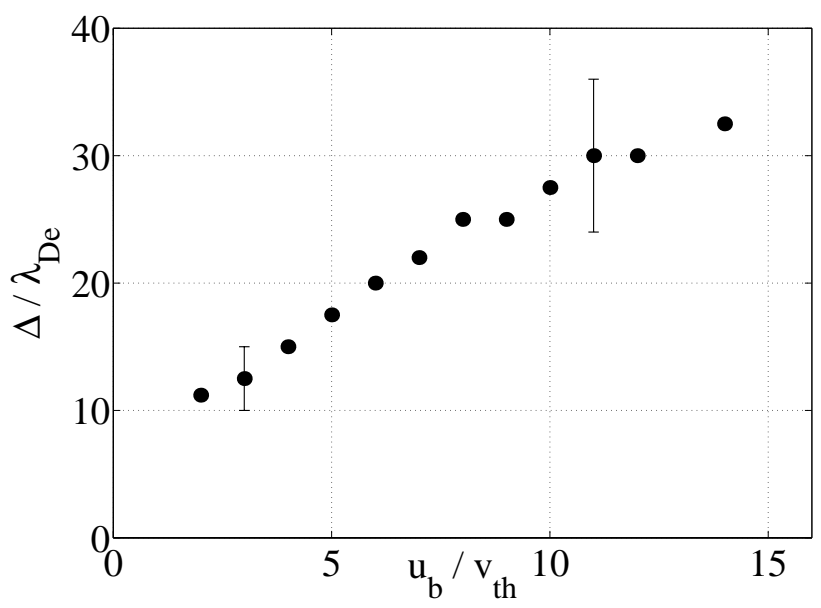

Fig. 6. Variation of the electron hole width, $\Delta$, for varying electron beam velocity. The uncertainty indicated arises predominantly from the scatter in magnitude among several holes being excited.

tion of the holes showed modest variation with $B$. The hole lifetime exceeded 100 electron plasma periods, at least with the assumption of immobile ions. With larger beam velocities, the magnetic field had a smaller effect. The study of Singh et al. (2000) used $\omega_{c e}=2 \omega_{p e}$ and also $\omega_{c e}=5 \omega_{p e}$. These results were later extended to cover a wider range of parameters (Singh et al., 2001a). Variations in the beam diameter had only minor effects on the hole evolution in our simulations, at least with the present parameters. In order to investigate the long time stability of electron holes, times $\gg \omega_{p i}^{-1}$, we find that the ion dynamics must be taken into account, in particular also because the injection of the electron beam violates the charge neutrality to build up low-frequency electric fields, which ultimately sets the ions in motion.

The velocity of the electron phase space vortices increased approximately with the injected beam velocity; the other parameters held constant. The formation time of the vortices also seemed to increase with the beam velocity, although it was more difficult to quantify this time scale. The fast electron holes (those with velocities much larger than $\sim 10 v_{t h}$ ) escaped from the simulation domain too fast to allow for an estimate of their lifetime. We would like to point out that holes with velocities much larger than the ion sound speed in magnetized plasmas can couple effectively only to ion cyclotron waves and lower-hybrid waves, where the latter coupling was observed by Singh et al. (2001b). This type of radiation is not found here due to the assumption of immobile ions. It is tempting to make a fast electron hole stationary in the computational reference frame by letting the background electron component move with a suitably chosen negative velocity, and reducing the beam velocity correspondingly. This has been tried, and indeed, it is then possible to also investigate these electron holes for extended simulation times. The boundary conditions for the two cases (stationary vs. moving background populations) are, however, no longer similar, and we find it inadvisable to compare the results from two different conditions, unless the spatial simulation domain is very large, namely larger than feasible with our computer resources.

\section{Ion phase space vortices}

The experiments described in Sect. 2 dealt with phenomena on short time scales, where the ion component could be considered immobile. Similar vortical structures were found numerically (Sakanaka, 1972; Pécseli et al., 1984) and experimentally (Pécseli et al., 1981, 1984) also in ion phase space, where now both electron and ion components participate in the plasma dynamics. In the experiments on ion phase space vortices, the evolution of the ion distribution function, and thereby, the phase space dynamics, could be measured directly by an ion energy analyzer (see also a summary by Pécseli, 1984). Other experiments later confirmed parts of these observations (e.g. the works by Chan et al., 1984), but investigated only space-time variations of plasma density and potential. Recent and more modern techniques have shown promise in detailed investigations of the ion phase space dynamics associated with the formation of ion holes (Skiff et al., 2001). Recent observations by Nakamura et al. (1999) also point out the formation and propagation of "ion holes", i.e. ion phase space vortices. These experiments were carried out under conditions similar to those in the studies by Pécseli et al. (1981) or Pécseli et al. (1984), but with larger electron to ion temperature ratios. All of these investigations were dealing with externally excited coherent phenomena. Evidence for the formation, propagation and subsequent decay of phase space vortices was also obtained in experiments carried out in an unmagnetized double-plasma device (Johnsen et al., 1985, 1987), where an ion beam was injected into a background plasma. A conditional sampling method was used for analyzing the space-time variation of the spontaneously generated fluctuations in plasma density. These studies differ from those previously mentioned by making a statistical analysis of the spontaneously generated fluctuations.

It is not evident that the conclusions from electron beam instabilities can be generalized to ion-ion beam instabilities right away, because in this latter case, the one-dimensional case is only weakly dispersive, and directional dispersion of ion beam generated waves is only significant for high beam velocities. One motivation for our study was to also investigate this question with the numerical plasma simulation to be described. (This foregoing discussion deals with the full conditions for the excitation of phase space vortices, and has little to do with the actual stability of an individual vortex; the appropriate stability analysis has been subject to investigations by, for instance, Schamel (1982) and Turikov (1984).) Also, the numerical study of Morse and Nielson (1969) refers to an initial condition, where the beams are present all over space; this is not the most relevant experimental situation. We wanted to study an experimentally, more realistic condition, where a beam is injected at the boundary of a pre- 
existing background plasma. The analysis is restricted to electrostatic phenomena occurring on time scales below the ion plasma frequency.

\subsection{Model discussions}

In the present case, we can assume that the electrons are isothermally Boltzmann distributed at all times (Børve et al., 2001). This simplification is obtained at the expense of a nonlinear Poisson equation. It is possible to generalize the model somewhat by allowing for deviations from the strict Boltzmann distribution. A useful model for this purpose was suggested by, for instance, Cairns et al. (1995); Mamun and Cairns (1996), and it leads to a Poisson equation of the form

$$
\begin{aligned}
& \nabla^{2} \Phi(\mathbf{r}, t)= \\
& \frac{e}{\varepsilon_{0}}\left[n_{0}\left(1-\beta \frac{e \Phi(\mathbf{r}, t)}{T_{e}}+\beta\left(\frac{e \Phi(\mathbf{r}, t)}{T_{e}}\right)^{2}\right) e^{e \Phi(\mathbf{r}, t) / T_{e}}\right. \\
& -n(\mathbf{r}, t)]
\end{aligned}
$$

in terms of the electron temperature $T_{e}$, the ion density $n$ and the electrostatic potential $\phi$, where the parameter $\beta$ measures the deviations from a Boltzmann distribution. The parameter is related to the electron energy distribution by $f(\epsilon)=\left(1+\alpha \epsilon^{2}\right) e^{-\epsilon} /(1+3 \alpha)$, with $\beta \equiv 4 \alpha /(1+3 \alpha)$ and $\epsilon$ being the normalized electron energy. A strict Boltzmann distribution is obtained trivially by $\alpha=0$. The model was originally proposed for a one-dimensional system, but here it is used in three dimensions. The argument is that given the present homogeneous magnetic field configuration with a low $\beta$ plasma, we can assume that the electrons are magnetized with a negligible Larmor radius, even though the ions can be weakly magnetized. In effect, we assume that the electron gas adjusts to changes in the plasma density and potential by flowing along magnetic field lines. The ideas proposed by Cairns et al. (1995) can be considered as representative for inclusion of a wider class of non-Maxwellian electron distributions (Summers and Thorne, 1991), which we will consider in a forthcoming study. Evidently, when $\alpha \neq 0$, we are dealing with a non-Maxwellian plasma, where the electron temperature cannot be properly defined. In this case, we let $T_{e}$ refer to the exponential multiplier $\exp \left(e \Phi(\mathbf{r}, t) / T_{e}\right)$ in Eq. (1). In terms of the "width" $\left\langle u^{2}\right\rangle \equiv \int u^{2} f(u) d u$ of the velocity distribution function in the B-parallel direction, we have $\left.\left\langle u^{2}\right\rangle=(1+15 \alpha) v_{t h}^{2} /(1+3 \alpha)\right)$, where $v_{t h}$ is the thermal velocity of a Maxwellian with temperature $T_{e}$ in the exponential multiplier in Eq. (1).

We would like to point out a constraint implied in the use of Eq. (1), which, in the general case, can be nontrivial. Thus, we note that overall charge conservation within the present model implies

$$
\begin{aligned}
& \int\left[n_{0}(t)\left(1-\beta \frac{e \Phi(\mathbf{r}, t)}{T_{e}}+\beta\left(\frac{e \Phi(\mathbf{r}, t)}{T_{e}}\right)^{2}\right) e^{e \Phi(\mathbf{r}, t) / T_{e}}\right. \\
& -n(\mathbf{r}, t)] d x d y d z=0,
\end{aligned}
$$

which, in general, determines $n_{0}(t)$ at all times for an unmagnetized plasma. In the magnetized case, with inhomogeneous plasmas having a density gradient perpendicular to the magnetic field, the expression has to be trivially modified. For the present simulations we found that the deviations in $n_{0}(t)$ from the initial unperturbed value were small.

The approach based on Eq. (1) has some obvious advantages; first, we avoid unphysical electron-ion mass ratios often employed to reduce computer requirements. Second, we can readily identify the specific ion phase space dynamics important for the observed time evolutions. The expense of the model is obviously that the electron phase space information is lost. This information would be crucial, for instance, for electron-ion two-stream instabilities and electron-hole interactions with ions (Saeki and Juul Rasmussen, 1991; Saeki and Genma, 1998). These and similar phenomena are excluded from our analysis by the choice of Eq. (1). We note that in case of high electron-ion temperature ratios, a parameter range exists where electron collisions are important, while ions can be considered collisionless. In this case, our basic assumption is easily justified. Since we want to be able to consider arbitrary length scales, we do not assume quasineutrality from the outset.

\subsection{Numerical results}

For our numerical studies we use the same code as described in Sect. 2.2, with the only complication here being a nonlinear Poisson equation (Pécseli et al., 1984; Børve et al., 2001). This equation is solved by a nonlinear multigrid method, which consists of a full multigrid schedule with an adaptive strategy that allows one to skip the coarse-grid correction if the specified accuracy is already reached on that grid. This schedule might be followed by some iterations of adaptive multigrid schemes in order to improve the accuracy (Wesseling, 1991). The relaxation method uses a Gauss-SeidelNewton iteration scheme.

As an illustration, we use the beam density to be one-half of the ion background density. The ion beam temperature is $T_{b}=0.1 T_{i}$, while the ion beam velocity is $3 v_{t i}$ in terms of the background ion thermal velocity. The electron temperature is $T_{e}=10 T_{i}$. In computational units, we have the background ion temperature to be $T_{i}=1$. The magnetic field intensity is chosen so that $\Omega_{c i} / \omega_{p i}=0.5$. The cross section of the ion beam is elliptical, with aspect ratio 0.22 . The major axis is $73 \%$ of the $x$-dimension of the simulation box, where we use $56 \times 56 \lambda_{D i}^{2}$ in the $(x, z)$-plane, and $368 \lambda_{D i}$ along the $y$-direction, which is parallel to $\mathbf{B}$.

In Fig. 7, we show the space time evolution of the electrostatic potential along the center line of the system for two cases: Boltzmann distributed electrons, where $\alpha=0$, and a case with significant deviations from this equilibrium distributions, with $\alpha=0.2$. Positions are measured along the $y$-axis in units of ion Debye lengths, $\lambda_{D i}$, using the temperature of the background ions, and time is measured in units of $\omega_{p i}^{-1}$, the inverse ion plasma frequency. The space-time evolution of the potential demonstrates the formation of a shock-like structure, with large amplitude, which propagates at a relatively high supersonic speed. The shock is trailed 

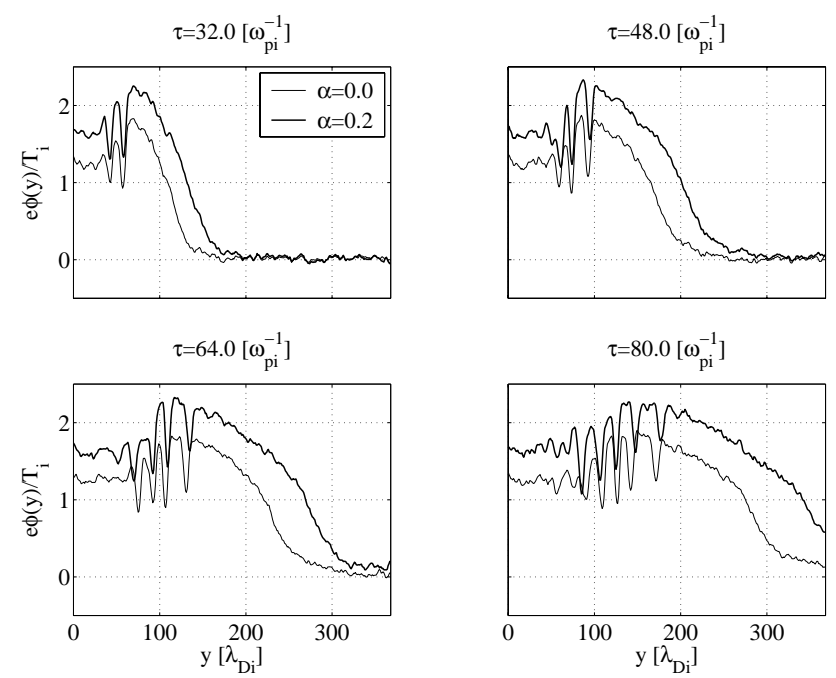

Fig. 7. Space-time evolution of the normalized electrostatic potential, $e \phi / T_{i}$, from a numerical simulation. The potential is sampled along the center axis of the simulation.

by a number of somewhat slower potential depletion, which we identify in the following as ion phase space vortices. The conspicuous effect of $\alpha \neq 0$ seems to be an increase in ion shock velocity. As for the ion holes trailing the shock, we see only modest differences between the two cases, and a typical hole width is of the order of $10-15 \lambda_{D i}$ in all cases.

Of course, the information on the ion density is also available from the simulations. We have not made any assumptions of quasi-neutrality in the basic model, and the density information is not trivially related to the potential. The difference between the two quantities is, however, not sufficient to justify a separate figure, and Fig. 7 can also be taken as a representative for the ion density variation, apart from a normalizing constant.

In order to establish a connection to the question of plasma stability, we show in Fig. 8 a numerical solution of the dispersion relation obtained for the relevant parameters, with $\alpha=0$. Our code for solving the dispersion relation assumes that parameters are entered for a physical condition, so we have chosen parameters relevant, for instance, for the FAST satellite (Ergun et al., 1998a, b), with densities $6 \cdot 10^{6} \mathrm{~m}^{-1}$ and magnetic fields $11 \cdot 10^{-6} \mathrm{~T}$. As an illustration, we took an electron temperature of $2 \mathrm{eV}$. Relative values for the beam and background plasma parameters are the same as in the simulations. With these parameters, the linearly, most unstable wavelength is approximately $5 \lambda_{D e}$, in terms of the electron Debye length. We note that a wide range of wave numbers is unstable. We recall here that given $\left(k_{\|}, k_{\perp}\right)$, there will be, at most, a finite, discrete set of solutions for frequencies corresponding to unstable solutions, while there are in general infinitely many damped solutions. Small "fringes" in the dispersion relation are due to competing roots. The most important observation in the present context may simply be that the plasma is linearly unstable (Pécseli and Trulsen, 1982; Pécseli et al., 1984).

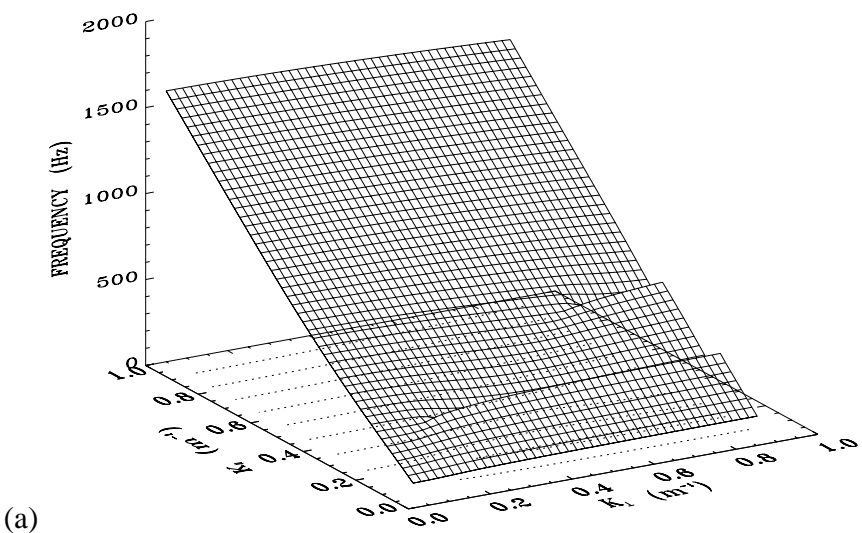

(a)

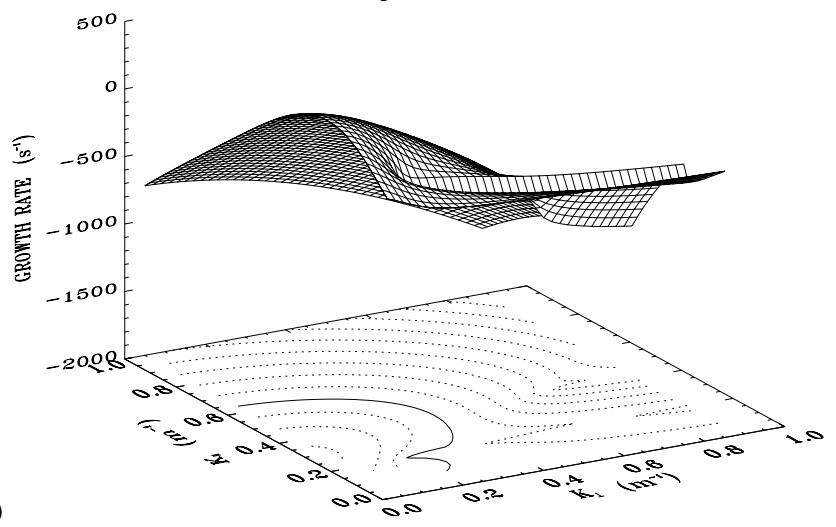

Fig. 8. Numerically obtained solution for the linear dispersion relation for the magnetized ion beam plasma. The real part of the frequency is shown in (a), the imaginary part in (b). The heavy line on the contour plot in (b) is the marginaly stable curve.

We have performed simulations with different beam velocities, and found that for low beam velocities, where the plasma is stable, no vortices are formed, although a weak shock may still develop. For very high ion beam velocities, the plasma will remain unstable, but in this case, for waves on the ion cyclotron branch (Michelsen et al., 1976). In this case, the linearly most unstable waves propagate with at an angle to the magnetic field (Michelsen, 1976), and no phase space vortices are formed (see also summary by Børve et al., 2001). The plasma is stable for small ion beam velocities below the sound speed, and it turns out that ion holes are being formed in a rather narrow "window" of ion beam velocities, and the variation of hole widths for varying ion beam velocities is rather modest (Børve et al., 2001).

The evolution in ion phase space corresponding to Fig. 7 is summarized in Figs. 9, 10 and 11. The figures are constructed as an average over a quadratic cross section of $56 \times 56 \lambda_{D i}^{2}$, perpendicular to the beam direction. In the numerical code, it is evidently possible to label the ions belonging to the background and the beam component, respectively. Thus, Fig. 9 illustrates the dynamics of the beam ions, where in this case, the ion phase space is spanned by the axis along the beam (and magnetic field) direction, and the velocity component in the same direction. We see very clearly the acceleration of the ions by the electric field in the shock, 

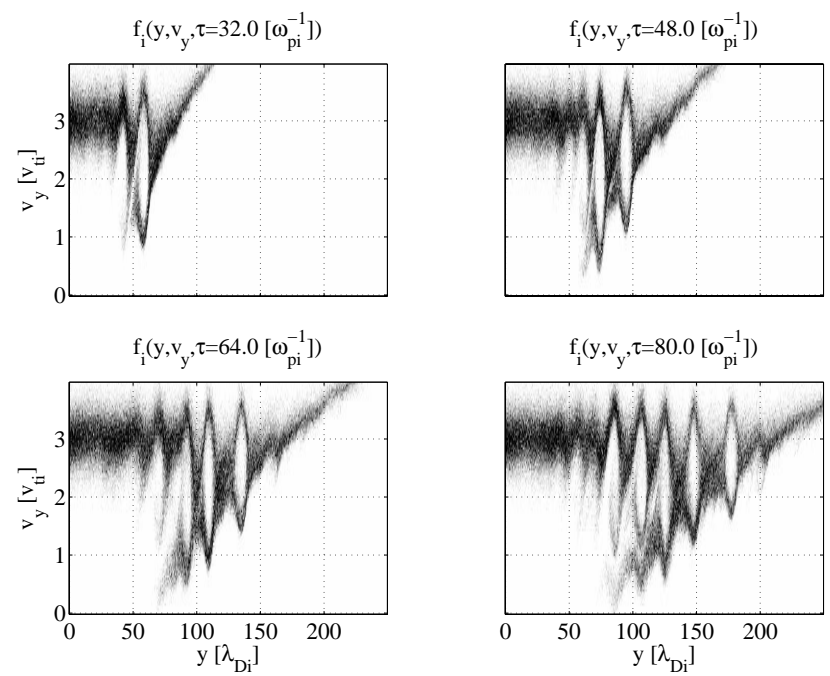

Fig. 9. Evolution in ion phase space of the beam ion component.
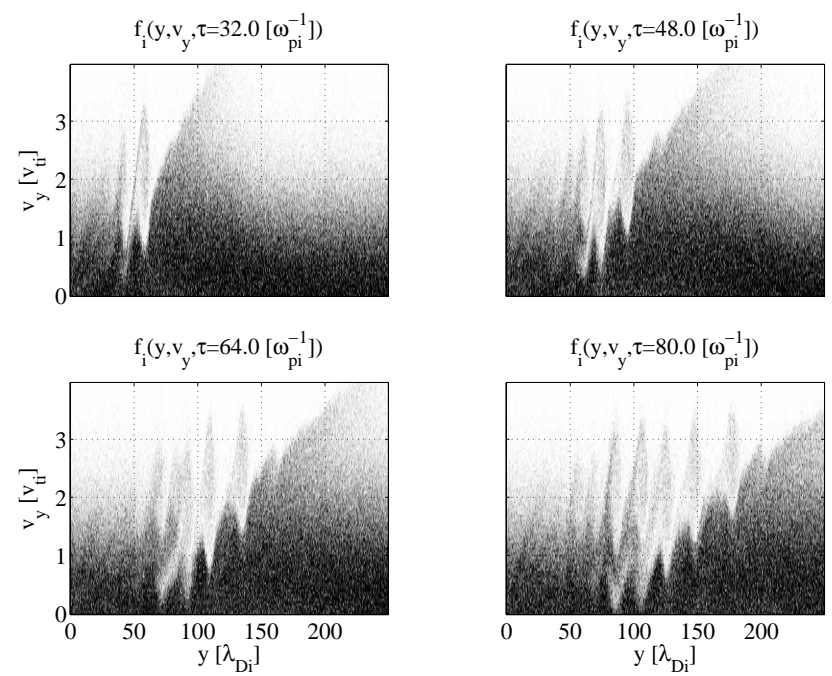

Fig. 10. Evolution of the background ion component in ion phase space.

followed by a number of ion phase space vortices, where this number increases with time. For the background ions, in the same phase space, as shown in Fig. 10, we note a temperature increase associated with the shock, and also here, we see some of the background ions being trapped in the phase space vortices. The phase space vortices form in the linearly, unstable ion-ion stream region behind the shock (Sakanaka, 1972; Pécseli and Trulsen, 1982; Pécseli et al., 1984). A typical width of the vortices is approximately $15 \lambda_{D i} \approx 5 \lambda_{D e}$, i.e. of the same order of magnitude as the linearly, most unstable wavelength (see Fig. 8), compatible with the observations of Pécseli et al. (1984). Significantly wider ion phase space vortices can develop late in the process, due to coalescence of smaller ones. The initial separation of the vortices is comparable to their width, but we see some of them separate slowly, due to a slight scatter in velocities.
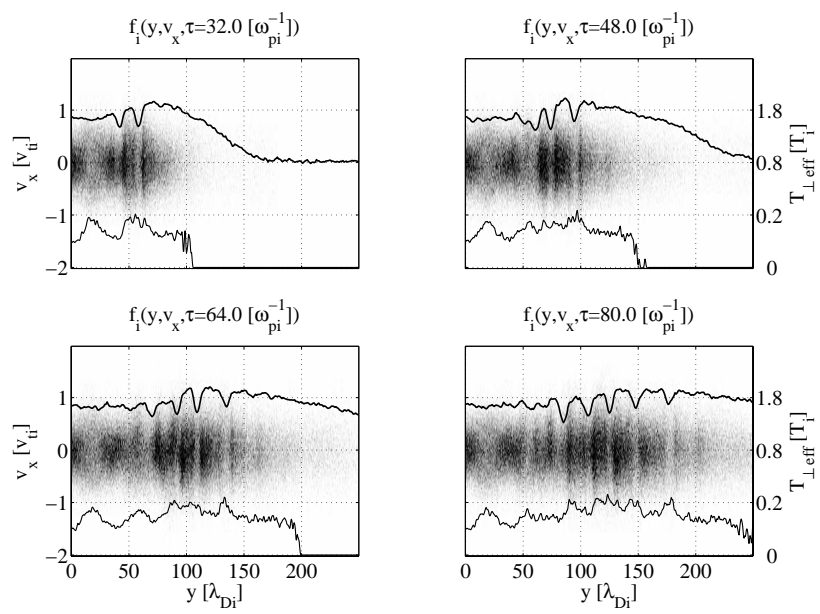

Fig. 11. Evolution in beam ion component in ion phase space composed by the spatial variation along the beam velocity ( $y$-axis) and the ion velocity component in the perpendicular direction. The solid line at the bottom gives the local effective temperature derived from the velocity distribution, with the corresponding axis to the right in the figure. The upper solid line shows the potential variation from Fig. 7 as a reference, in arbitrary units.

The ambipolar electric field associated with the shock is due to the electron thermal pressure, which gives rise to an expansion of the electron component, implying a small imbalance in the relative electron and ion densities in the Poisson Eq. (1). This electric field accelerates the ions in front of the shock, as seen in Figs. 9 and 10. In particular, by considering the background ion population in Fig. 10, we note that the shock acts as a "snowplow", accelerating background ions to velocities exceeding that of the average injected beam velocity. Details of the shock formation associated with injected ion beams have been discussed by Ikezi et al. (1973). If $\alpha$ is increased from the zero value corresponding to the Maxwellian electron distribution, then the number of energetic electrons is increased, and the ambipolar electric field is increasing, i.e. the potential level is enhanced, as seen in Fig. 7. This enhanced electric field gives rise to a corresponding increase in the velocity of the shock front, as is also apparent in Fig. 7.

Even more interesting is the ion beam evolution in phase space spanned by the $y$-axis and the velocity component in the direction perpendicular to this (see Fig. 11). The solid lines on these figures show the local effective ion beam temperature in the $v_{x}$-direction, obtained by calculating an effective perpendicular ion beam temperature

$T_{\perp \text { eff }} \equiv \frac{\int v_{x}^{2} f\left(y, v_{x}, \tau\right) d v_{x}}{\int f\left(y, v_{x}, \tau\right) d v_{x}}$.

The ragged portion of the figure at the very end is due to a small number of simulation particles there. We find a noticeable ion heating (by almost a factor of 2) associated with the formation of the ion vortices. This effect is also observed 
for the case where $\alpha=0$, but it is less pronounced then. By comparison with the potential variations, we find a clear one-to-one correspondence between the striations in Fig. 11 and the positions of the vortices. To make this feature more clear, we also inserted in Fig. 11 the potential variation from Fig. 7 in arbitrary scale, having the corresponding zero-line to be the same as for the velocity. As expected, we found the regions with a local decrease in phase space density corresponding to the potential minima. For fluctuations being close to quasi-neutral, i.e. $e \phi / T_{e} \sim n / n_{0}$, the potential minimum corresponds to the local density minimum. The local transverse beam temperature enhancement corresponds, on the other hand, mainly to the regions between the ion holes. In some cases, we can identify the position of the local transverse temperature enhancement to be the "flank" of the ion hole facing the ion beam injection. Since the electrostatic ion holes propagate with almost no change in shape, they cannot energize charged particles, and the increase in perpendicular beam temperature is, therefore, due to scattering of the parallel velocity. In the present case, it is the directed ion beam energy which, by scattering, is transformed to an increase in the effective ion temperature perpendicular to $\mathbf{B}$.

In order to investigate whether this transverse ion beam heating was due to a "bulk" or to a "tail" heating, we calculated the local excess or flatness factor (Cramér, 1946), $K$, of the distribution at selected times, for varying spatial positions. We found that the kurtosis was slightly negative, $K \sim\{-0.1,-0.2\}$, implying that we have a slight under-representation of fast particles, as compared to the Maxwellian distribution, where $K=0$. Intuitively, this seems reasonable, since fast particles have a larger probability of simply passing through the three-dimensional potential distribution associated with the phase space vortex. Consequently, the slower beam ions will, in comparison, contribute slightly more to the transverse distribution.

The coupling of the vortices to the beam is stronger than to the background, because the ion vortex has a velocity closer to the beam velocity for the present parameters. We note that the observed structures are fully three-dimensional, having an electric field component along, as well as perpendicular to, the magnetic field lines. As an illustration, we show in Fig. 12 the electrostatic potential in a quadrant of the simulation space. The gaps in the figure indicate the potential depletion associated with the ion holes. Inspection of a figure corresponding to Fig. 11 for the background ions shows only a small change in the $\mathbf{B}_{\perp}$ ion temperature, and this figure need not be presented here.

As discussed by Daldorff et al. (2001), the ion phase space vortices seem to have some internal degrees of freedom, which are manifested by an oscillatory motion, or vibration, of the structure in such a way that the phase space volume of the vortex is conserved. This can also be observed in the 2.5dimensional studies of Børve et al. (2001). These features seem to have their counterparts in the dynamics of electron holes, as discussed by Oppenheim et al. (1999), Singh et al. (2000) and Newman et al. (2001).

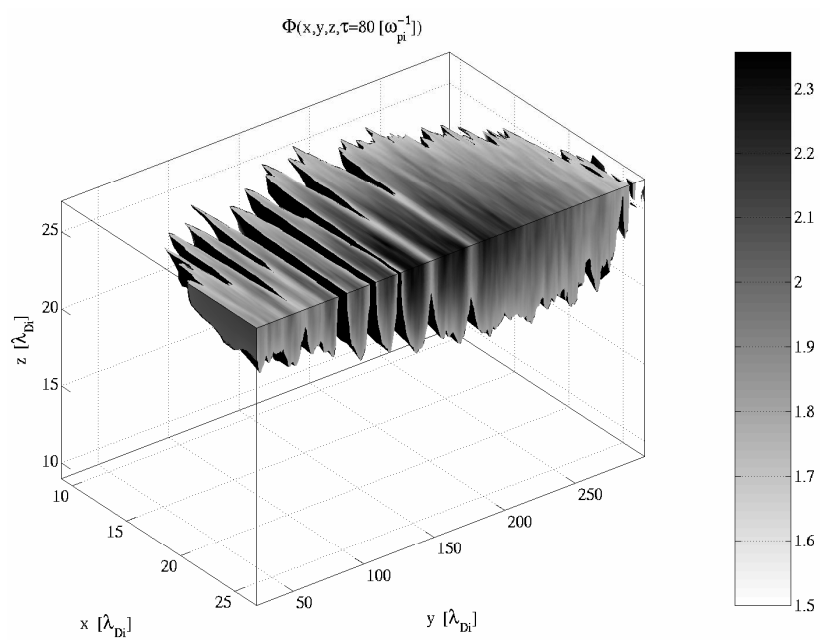

Fig. 12. The spatial variation of the electrostatic potential at a time $\tau=80 \omega_{p i}^{-1}$ is shown in one quadrant of the simulation domain. Levels for the potential $e \phi / T_{i}$ are shown with grey-scale. Only regions where the normalized potential levels are above 1.5 are shown. The maximal values are approximately 2.4 in normalized units.

\section{Relation to space observations}

We believe the present results can be relevant for interpreting data from the ionospheres and magnetospheres of magnetized planets, such as the Earth. Solitary structures have been observed on spacecraft (see summary of, for instance, Maelkki et al., 1993) and Eriksson et al. (1997). The origin of these structures was expected to be a nonlinear instability, driven by weak currents, but the data actually speak strongly against this interpretation by showing no preferred direction for the propagation of the observed structures (Mälkki et al., 1993). These observations were made in a plasma environment where $n_{0} \approx 10^{6}-10^{7} \mathrm{~m}^{-3}$ and $B \approx 4 \mu \mathrm{T}$, giving a proton plasma frequency $\omega_{p i} /(2 \pi) \approx 470 \mathrm{~Hz}$, and a gyrofrequency of $60 \mathrm{~Hz}$, implying $\omega_{p i} / \Omega_{c i} \approx 8$. We do not find the interpretation of these satellite observations to be self evident, but in case that the observed potential wells are to be associated with ion phase space vortices, our numerical results would indicate that they are rapidly damped. This observation implies, in turn, that they have to be found in the near vicinity of the region where they were created. The observations are consistent with a lifetime of the order of 0.01 s. An estimate of the bounce period of an ion can be obtained as $t_{B} \sim L \sqrt{M /\left(e\left|\phi_{0}\right|\right)}$. With the observed values of widths $L \approx 60 \mathrm{~m}$ and peak potential depletions $\phi_{0} \approx 4 \mathrm{~V}$, we have $t_{B} \sim 3 \cdot 10^{-3} \mathrm{~s}$, which indicates that the lifetimes might typically be 3 ion bounce times (the value for $\phi_{0}$ is an upper estimate). This result is compatible with our observations experimentally (Pécseli et al., 1981, 1984; Johnsen et al., 1985, 1987), as well as numerically (Børve et al., 2001).

Indications of the presence of electron phase space holes were found in the data from the FAST satellite (Ergun et al., 1998a, b), and these are particularly interesting due to their 
relatively large amplitudes, i.e. $\left|e \phi_{0}\right| \sim T_{e}$. For this case, a magnetic field of approximately $11 \cdot 10^{-6} \mathrm{~T}$ was reported (Ergun et al., 1998b), giving $\omega_{c e} \approx 2 \cdot 10^{6} \mathrm{~s}^{-1}$, and a plasma density approximately $6 \cdot 10^{6} \mathrm{~m}^{-3}$, implying $\omega_{p e} \approx 1.4 \cdot 10^{5}$ $\mathrm{s}^{-1}$, giving $\omega_{c e} / \omega_{p e} \geq 1$. For these conditions, we would expect electron phase space vortices to be relatively stable.

Observations of electrostatic solitary structures in the magnetotail of the Earth have been reported from data obtained by the GEOTAIL satellite (Krasovsky et al., 1997; Omura et al., 1999). It is plausible that these can be interpreted in terms of phase space structures, although a significant amount of work remains to be done concerning the derivation of an actual spatial structure, which in the experiments has to be deduced from a time varying signal obtained from the potential difference between two probes with large separation, on the moving spacecraft. Relevant observations were also made by the POLAR spacecraft (Franz et al., 1998), where structures associated with a local net positive charge were found. Also, other observations from the POLAR satellite show isolated electric field structures (Mozer et al., 1997). Numerical studies carried out by Miyake et al. (1998) were aimed, in particular, at the interpretation of the GEOTAIL data. It will be interesting to see whether the data from the CLUSTER satellites will reveal signatures of plasma solitary structures. In this case, the spatial resolution of these satellites will offer a good possibility for determining conditions for the occurrence of such structures.

Our results also focus on the curious preference for small amplitude narrow structures being observed by many spacecraft (i.e. amplitudes giving a potential energy at the peak potential $\phi_{0}$ much less than the thermal energy, $e\left|\phi_{0}\right| \ll T$, and widths comparable to the Debye lengths). In contrast, our experimental, as well as numerical observations are made for large amplitude and relatively wide structures, for electron as well as ion holes. Obviously, in an experiment, the consideration of signal-to-noise ratios will emphasize large amplitude structures, but we find their absence in space observations curious. There is, in principle, no lower limit to the amplitude of phase space vortices, but we anticipate that their formation time can be large for small amplitudes. This latter conjecture is justified if we, as an estimate for the formation time, use the bounce period $\tau_{B} \sim \ell \sqrt{m /\left(\left|q \phi_{0}\right|\right)}$ of a charged particle with mass $m$ and charge $q$, in a potential well of width $\ell$ and peak amplitude $\phi_{0}$. The argument is applicable for the case where the phase space structures form by trapping in a potential well formed by some disturbance, as in the experiment of Lynov et al. (1979). For phase space holes formed by, for instance, beam instabilities, the formation time may be shorter.

\section{Conclusions}

In the present paper, we studied the formation and evolution of electron, as well as ion phase space vortices, and also reviewed some previous experiments by presenting some hitherto unpublished results. The electron holes were excited ex- ternally by an electric pulse accelerating the electrons in a localized spatial region. The space-time evolution of these structures was then followed. By inlet of a neutral gas component, it was, in this experiment, explicitly demonstrated that phase space structures are extremely sensitive to collisions. These observations were illustrated by Saeki et al. (1979) and Lynov et al. (1979). Due to the strong, externally imposed magnetic field, the electron holes propagated as in an ideal one-dimensional system, and we saw no indication of any decay or finite lifetimes. The formation and evolution of electron phase space vortices was also illustrated by a numerical simulation, assuming immobile ions (see Figs. 4 and 5 ). This beam excitation is different from that used in the experiments, but the resulting phase space vortices have quite the same dynamics.

The present studies of ion phase space vortices were based on a fully three-dimensional numerical particle simulation based on a previous 2.5-dimensional code, also described by Guio et al. (2001). The vortices were generated by an ion beam injected into a stationary background plasma. We observed the formation of BGK-type equilibria for all values of the magnetic field, also for $B=0$, but found that the lifetime of the structures increased rapidly with increasing magnetic field intensity. It thus seems plausible that the type of twodimensional structures in unmagnetized plasmas anticipated by Kato (1976) and Ishibashi and Kitahara (1992) can be formed, but that they are inherently unstable. Our numerical results indicate that, for the plasma parameter range relevant here, the structures are relatively stable in two as well as three spatial dimensions, when the transverse length scale significantly exceeds the ion Larmor radius and simultaneously $\Omega_{c i}>\omega_{p i} / 2$. As a new feature of our simulations, we introduced here deviations from the strict Boltzmann electron distribution by using a standard model (Mamun and Cairns, 1996). The overall consequence of a deviation from strictly Boltzmann distributed electrons is found to be an increase in the electrostatic fields, which is, after all, to be expected from an enhanced energetic component accounted for by $\alpha \neq 0$. On the other hand, it is also fair to add that the effect of a small energetic electron population is minor, as far as the ion phase space vortices are concerned, but it may be important for ion acoustic shock dynamics.

Visual inspection of the representation of phase space vortices in Figs. 5, 9, and 10 show a noticeable similarity, but this is to some extent deceiving. Some of the parameter variations are basically different, where we, in particular, emphasize that electron vortices seem to be formed for all beam velocities exceeding $\sim 2 v_{t h}$, while the ion vortices are observed only for a restricted interval of ion beam velocities.

Phase space vortices seem to be a generic feature of the saturated stage of beam plasma instabilities (Kako et al., 1971; Pécseli and Trulsen, 1982; Pécseli et al., 1984), at least in one spatial dimension, indicating that this saturated stage can be considered as a relatively low-dimensional system, described by a random superposition of such coherent structures, together with a low level noise component (KofoedHansen et al., 1989). Phase space vortices can, however, 
be excited by other means as well, such as short particle bursts, for instance, and not necessarily by beam instabilities (Pécseli, 1984). As the laboratory results summarized in Sect. 2 demonstrate, an "impulse-like" perturbation can develop such vortices, provided that the amplitude is sufficiently large. In this case, the vortex formation appears as a result of particle trapping in the combined plasma and externally imposed potentials. It can be argued that neither the formation nor the lifetime of phase space vortices are understood in depth, although for different reasons. The formation by beam instabilities is well understood, but this is not the only way phase space vortices are formed, as mentioned. Concerning the vortex lifetime, the problem is basically different. First of all, it is obvious that an initial excitation cannot evolve into an exact time-stationary solution for the Vlasov equation. We can, however, consider the resulting structure as such an exact solution with some added perturbation, and ask whether during the future time evolution it remains close to the exact solution (implying stability) or deviates from it (corresponding to instability). Some investigation of the stability of phase space structures has been carried out (Schamel, 1982; Turikov, 1984; Schamel, 1986), but the problem cannot be considered as fully resolved, in particular not as far as the fully three-dimensional evolution is concerned. The main concern of the present discussion is isolated phase space structures, but it may be appropriate to mention that the stability periodic fully nonlinear (onedimensional) BGK-solutions were studied much earlier by, for instance Goldman (1970).

Phase space vortices are interesting examples of self organization in highly dynamic, nonlinear systems. By forming well-defined entities, these phase space structures allow for the saturated stage of, for instance, beam instabilities to be described by a modest number of variables, such as positions, widths and amplitudes of the structures.

Acknowledgements. This work was in part supported by the Norwegian Research Council. The laboratory experiments summarized in Sect. 2 would not have been possible without the expert assistance of M. Nielsen, B. Reher and K. Weisberg. We thank V. A. Turikov for many valuable discussions on electron phase space vortices. The original numerical simulations supporting the interpretation of the experimental results were made by him.

\section{References}

Berk, H. L., Nielsen, C. E., and Roberts, K. V.: Phase space hydrodynamics of equivalent nonlinear systems: Experimental and computational observations, Phys. Fluids, 13, 980-995, 1970.

Bernstein, I. B., Green, J. M., and Kruskal, M. D.: Exact nonlinear plasma oscillations, Phys. Rev., 108, 546-550, 1957.

Birdsall, C. K. and Langdon, A. B.: Plasma Physics via Computer Simulation, McGraw-Hill, New York, 1985.

Børve, S., Pécseli, H. L., and Trulsen, J.: Ion phase-space vortices in 2.5-dimensional simulations, J. Plasma Phys., 65, 107-129, 2001.

Cairns, R. A., Mamun, A. A., Bingham, R., Boström, R., Dendy, R. O., Nairn, C. M. C., and Shukla, P. K.: Electrostatic soli- tary structures in non-thermal plasmas, Geophys. Res. Lett., 22, 2709-2712, 1995.

Chan, C., Cho, M., Hershkowitz, N., and Intrator, T.: Laboratory evidence for ion-acoustic-type double layers, Phys. Rev. Lett., 52, 1782-1785, 1984.

Cramér, H.: Mathematical Methods of Statistics, Princeton University Press, 1946.

Daldorff, L. K. S., Guio, P., Børve, S., Pécseli, H. L., and Trulsen, J.: Ion phase space vortices in 3 spatial dimensions, Europhys. Lett., 54, 161-167, 2001.

Ergun, R. E., Carlson, C. W., McFadden, J. P., Mozer, F. S., Delory, G. T., Peria, W., Chaston, C. C., Temerin, M., Roth, I., Muschetti, L., Elphic, R., Strangeway, R., Pfaff, R., Catell, C. A., Klumpar, D., Shelley, E., Peterson, W., Moebius, E., and Kistler, L.: FAST satellite observations of large-amplitude solitary structures, J. Geophys. Res., 25, 2041-2044, 1998a.

Ergun, R. E., Carlson, C. W., McFadden, J. P., Mozer, F. S., Muschietti, L., and Roth, I.: Debye-scale plasma structures associated with magnetic-field-aligned electric fields, Phys. Rev. Lett., 81, 826-829, 1998b.

Eriksson, A. I., Mälkki, A., Dovner, P.-O., Boström, R., Holmgren, G., and Holback, B.: A statistical survey of auroral solitary waves and weak double layers 2 . measurement accuracy and ambient plasma density, J. Geophys. Res., 102, 11 385-11398, 1997.

Franz, J. R., Kintner, P. M., and Pickett, J. S.: POLAR observations of coherent electric field structures, Geophys. Res. Lett., 25, 1277-1280, 1998.

Goldman, M. V.: Theory of stability of large periodic plasma waves, Phys. Fluids, 13, 1281-1289, 1970.

Guio, P., Børve, S., Pécseli, H. L., and Trulsen, J.: Low frequency waves in plasmas with spatially varying electron temperature, Ann. Geophysicae, 18, 1613-1622, 2001.

Iizuka, S. and Tanaca, H.: Evolution of electron hole and electron wave pulses in a single ended magnetoplasma, Phys. Fluids, 29, 4035-4039, 1986.

Ikezi, H., Kamimura, T., Kako, M., and Lonngren, K. E.: Laminar electrostatic shock waves generated by an ion beam, Phys. Fluids, 16, 2167-2175, 1973.

Ishibashi, N. and Kitahara, K.: Two-dimensional Bernstein-GreeneKruskal solution, J. Phys. Soc. Jpn., 61, 2795-2804, 1992.

Johnsen, H., Pécseli, H. L., and Trulsen, J.: Conditional eddies, or clumps, in ion-beam-generated turbulence, Phys. Rev. Lett., 55, 2297-2300, 1985.

Johnsen, H., Pécseli, H. L., and Trulsen, J.: Conditional eddies in plasma turbulence, Phys. Fluids, 30, 2239-2254, 1987.

Kako, M., Taniuti, T., and Watanabe, T.: Hole equilibria in a quasicold plasma, J. Phys. Soc. Jpn., 31, 1820-1829, 1971.

Karpman, V. I., Lynov, J. P., Michelsen, P., Pécseli, H. L., Juul Rasmussen, J., and Turikov, V. A.: Modification of plasma solitons by resonant particles, Phys. Rev. Lett., 43, 210-214, 1979.

Kato, K.: Two-dimensional and three-dimensional BGK waves, J. Phys. Soc. Jpn., 41, 1050-1053, 1976.

Kofoed-Hansen, O., Pécseli, H. L., and Trulsen, J.: Coherent structures in numerically simulated plasma turbulence, Phys. Scripta, 40, 280-294, 1989.

Krasovsky, V. L., Matsumoto, H., and Omura, Y.: BernsteinGreene-Kruskal analysis of electrostatic solitary waves observed with Geotail, J. Geophys. Res., 102, 22 131-22 139, 1997.

Lynov, J. P., Michelsen, P., Pécseli, H. L., Rasmussen, J. J., Saeki, K., and Turikov, V.: Observations of solitary structures in a magnetized, plasma loaded waveguide, Phys. Scripta, 20, 
$328-335,1979$.

Lynov, J. P., Michelsen, P., Pécseli, H. L., and Rasmussen, J. J.: Interaction between electron holes in a strongly magnetized plasma, Phys. Lett., 80A, 23-25, 1980.

Lynov, J. P., Michelsen, P., Pécseli, H. L., Rasmussen, J. J., and Sørensen, H.: Phase-space models for solitary electron holes, Phys. Scripta, 31, 596-605, 1985.

Mälkki, A., Eriksson, A. I., Dovner, P.-O., Boström, R., Holback, B., Holmgren, G., and Koskinen, H.: A statistical survey of Auroral solitary waves and weak double layers 1 . Occurrence and net voltage, J. Geophys. Res., 98, 15 521-15 530, 1993.

Mamun, A. A. and Cairns, R. A.: Stability of solitary waves in magnetized non-thermal plasma, J. Plasma Phys., 56, 175-185, 1996.

Michelsen, P.: Stability limits of the ion beam excited electrostatic ion cyclotron instability, Phys. Fluids, 19, 337-339, 1976.

Michelsen, P., Pécseli, H. L., Juul Rasmussen, J., and Sato, N.: Unstable electrostatic ion cyclotron waves excited by an ion beam, Phys. Fluids, 19, 453-456, 1976.

Miyake, T., Omura, Y., Matsumoto, H., and Kojima, H.: Twodimensional computer simulations of electrostatic solitary waves observed by Geotail spacecraft, J. Geophys. Res., 103, 11 841$11850,1998$.

Morse, R. L. and Nielson, C. W.: One-, two-, and three-dimensional numerical simulation of two-beam plasmas, Phys. Rev. Lett., 23, 1087-1090, 1969.

Motley, R. W.: Q Machines, Academic Press, New York, 1975.

Mozer, F. S., Ergun, R., Temerin, M., Cattell, C., Dombeck, J., and Wygant, J.: New features of time domain electric-field structures in the auroral acceleration zone, Phys. Rev. Lett., 79, 1281-1284, 1997.

Nakamura, Y., Bailung, H., and Shukla, P. K.: Observation of ionacoustic shocks in a dusty plasma, Phys. Rev. Lett., 83, 1602 $1605,1999$.

Newman, D. L., Goldman, M. V., Spector, M., and Perez, F.: Dynamics and instability of electron phase-space tubes, Phys. Rev. Lett., 86, 1239-1242, 2001.

Omura, Y., Kojima, H., Miki, N., Mukai, T., Matsumoto, H., and Anderson, R.: Electrostatic solitary waves carried by diffusive electron beams observed by the Geotail spacecraft, J. Geophys. Res., 104, 14627-14 637, 1999.

Oppenheim, M., Newman, D. L., and Goldman, M. V.: Evolution of electron phase-space holes in a $2 \mathrm{~d}$ magnetized plasma, Phys. Rev. Lett., 83, 2344-2347, 1999.

Pécseli, H. L.: Electron and ion phase space vortices, in: Proceedings from the Second Symposium on Plasma Double Layers and Related Topics, (Eds) Schrittwieser, R. and Eder, G., University of Innsbruck, Studia, Innsbruck, 81-117, 1984.

Pécseli, H. L.: Ion phase-space vortices and their relation to small amplitude double layers, Laser Part. Beams, 5, 211-217, 1987.

Pécseli, H. L. and Trulsen, J.: Nonlinear evolution of the ion-ion beam instability, Phys. Rev. Lett., 48, 1355-1358, 1982.

Pécseli, H. L., Trulsen, J., and Armstrong, R.: Experimental observation of ion phase-space vortices, Phys. Lett., 81A, 386-390, 1981.

Pécseli, H. L., Trulsen, J., and Armstrong, R.: Formation of ion phase-space vortexes, Phys. Scripta, 29, 241-253, 1984.

Roberts, K. V. and Berk, H. L.: Nonlinear evolution of the twostream instability, Phys. Rev. Lett., 19, 297-300, 1967.

Saeki, K.: Electron plasma wave shocks in a collisionless plasma, J. Phys. Soc. Jpn., 35, 251-257, 1973.

Saeki, K. and Genma, H.: Electron-hole disruption due to ion motion and formation of coupled electron hole and ion-acoustic soliton in a plasma, Phys. Rev. Lett., 80, 1224-1227, 1998.

Saeki, K. and Ikezi, H.: Electron-plasma-wave shocks in a bounded plasma, Phys. Rev. Lett., 29, 253-255, 1972.

Saeki, K. and Juul Rasmussen, J.: Stationary solution of coupled electron hole and ion soliton in a collisionless plasma, J. Phys. Soc. Jpn., 60, 735, 1991.

Saeki, K., Michelsen, P., Pécseli, H. L., and Rasmussen, J. J.: Formation and coalescence of electron solitary holes, Phys. Rev. Lett., 42, 501-504, 1979.

Sakanaka, P. H.: Beam-generated collisionless ion-acoustic shocks, Phys. Fluids, 15, 1323-1327, 1972.

Schamel, H.: Theory of electron holes, Phys. Scripta, 20, 336-342, 1979.

Schamel, H.: Linear modes in the presence of solitary ion holes, Phys. Lett., 89A, 280-282, 1982.

Schamel, H.: Electron holes, ion holes and double layers, Phys. Reports, 140, 161-191, 1986.

Singh, N. and Schunk, R. W.: Plasma response to the injection of an electron beam, Plasma Phys. Contr. Fusion, 859-890, 1984.

Singh, N., Loo, S. M., Wells, B. E., and Deverapalli, C.: Threedimensional structure of electron holes driven by an electron beam, Geophys. Res. Lett., 27, 2469-2472, 2000.

Singh, N., Loo, S. M., and Wells, E.: Electron hole structure and its stability depending on plasma magnetization, J. Geophys. Res. 106, 21 183-21 198, 2001a.

Singh, N., Loo, S. M., and Wells, E.: Electron hole as an antenna radiating plasma waves, Geophys. Res. Lett., 28, 1371-1374, 2001b.

Skiff, F., Bachet, G., and Doveil, F.: Ion dynamics in nonlinear electrostatic structures, Phys. Plasmas, 8, 3139-3142, 2001.

Summers, D. and Thorne, R. M.: The modified plasma dispersion function, Phys. Fluids B, 3, 1835-1847, 1991.

Turikov, V.: Electron phase space holes as localized BGK solutions, Phys. Scripta, 30, 73, 1984.

Wesseling, P.: An Introduction to Multigrid Methods, John Wiley \& Sons Ltd., 1991. 